\title{
Misturas NR/SBR: Influência da Composição e do Modo de Preparação Sobre Propriedades Mecânicas e Reométricas
}

\author{
Hérlon M. B. Abreu Junior, Regina C. R. Nunes, Leila L. Y. Visconte \\ Instituto de Macromoléculas Professora Eloisa Mano, UFRJ
}

\begin{abstract}
Resumo: Aplicações de produtos à base de borracha diversas vezes requerem um conjunto de propriedades as quais, geralmente, não são fornecidas por um único elastômero. Dessa forma, um dos recursos utilizados é a mistura de dois ou mais polímeros para obtenção de novas propriedades. Neste caso, os demais ingredientes que são adicionados, normalmente distribuem-se de forma irregular, dependendo de sua afinidade com cada uma das borrachas. $\mathrm{O}$ grau de dispersão de cada um desses ingredientes em cada fase elastomérica irá então influenciar a velocidade de cura e o grau de vulcanização e, certamente, terá conseqüências nas propriedades do produto final. Neste trabalho, a borracha natural (NR) foi misturada ao elastômero de butadieno-estireno (SBR) nas proporções de 75:25 e 50:50, em massa. As composições foram preparadas empregandose quatro maneiras distintas para a incorporação dos aditivos. Os resultados das propriedades mecânicas mostraram que, apesar da utilização de uma mesma formulação, o modo de preparo tem grande influência sobre as propriedades. No caso da mistura NR/SBR as melhores propriedades são obtidas quando é favorecida a vulcanização da borracha que, isoladamente, apresenta propriedades inferiores.
\end{abstract}

Palavras-chave: Borracha natural, misturas elastoméricas, propriedades mecânicas.

\section{NR/SBR Mixtures: Influence of Composition and Preparation Mode on the Mechanical and Rheological Properties}

Abstract: Frequently a set of properties required for a given application cannot be accomplished by one elastomer only and, thus, mixtures of two or more polymers must be employed. In these cases, the other ingredients added are normally dispersed in a non homogeneous way, depending on their affinity to each polymer. The degree of dispersion each additive experiences in each elastomeric phase will influence the rate and the degree of vulcanization and will certainly have consequences on the properties of the final product. In this work, natural rubber (NR) was mixed with styrene-butadiene elastomer (SBR) in 75:25 and 50:50 w/w ratios. The compositions were prepared in a two-roll mill, by introducing the additives according to four different modes. The results of mechanical properties show that, although having been prepared following the same formulation, the properties of these mixtures are influenced by the preparation mode. For NR/SBR blends, the best properties are achieved when one favours the vulcanization of the rubber with inferior properties.

Keywords: Natural rubber, rubber blends, mechanical properties.

\section{Introdução}

Nas últimas décadas vem crescendo significativamente o interesse em se produzir e aperfeiçoar novos materiais através da mistura de polímeros comercialmente disponíveis, com propriedades complementares ${ }^{[1]}$, ao invés de sintetizar novos polímeros. Essa estratégia é relativamente simples e comercialmente atrativa, quando comparada com a síntese.

A possibilidade de misturar dois ou mais tipos de polímeros é uma técnica vantajosa, uma vez que permite o desenvolvimento de novos materiais com propriedades superiores àquelas apresentadas pelos constituintes da mistura isoladamente ${ }^{[2]}$.

Um elastômero é misturado a outro por três razões principais: para melhorar as propriedades do elastômero original, melhorar o seu processamento e/ou baixar custos. As propriedades das misturas poliméricas dependem principalmente da miscibilidade entre os dois polímeros. As misturas podem ser heterogêneas ou homogêneas. Nas misturas homogêneas, as propriedades são afetadas pelas interações entre os componentes, enquanto para misturas heterogêneas, o fenômeno interfacial e a natureza flexível ou rígida das fases são importantes. Portanto, a maioria das misturas de borracha exibem propriedades inferiores às esperadas, em decorrência da incompatibilidade entre os componentes ${ }^{[3]}$.

Existem várias maneiras de se preparar uma mistura de elastômeros, sendo a mecânica a mais utilizada. Em qualquer sistema bifásico, existe a possibilidade de reações

Autor para correspondência: Leila L. Y. Visconte, Universidade Federal do Rio de Janeiro, Caixa Postal 68525, CEP: 21945-970, Rio de Janeiro, RJ, Brasil. 
químicas ocorrerem a diferentes taxas em cada uma das fases. Uma complicação adicional ocorre durante a vulcanização das misturas uma vez que os componentes elastoméricos que formam as fases também são reagentes. Portanto, a diferença entre as taxas das reações pode fazer surgir, em ambas as fases, uma diferença de concentração de um ou mais reagentes e/ou uma diferença de reatividade das duas fases com os agentes de cura. A diferença na concentração dos reagentes pode surgir, em cada uma das fases, em conseqüência da solubilidade preferencial dos agentes de cura ${ }^{[1]}$.

Neste trabalho foram empregadas duas borrachas muito comuns, cujos comportamentos em relação à resistência à tração são bem diferentes. A borracha natural (NR) tem excelente resistência à tração, mesmo na ausência de cargas reforçadoras como o negro de fumo, por ser cristalizável sob tração. A borracha de butadieno-estireno (SBR) não é cristalizável e apresenta propriedades mais modestas sendo, no entanto, a de mais baixo custo e, portanto, a mais utilizada para aplicações gerais em que não se necessita de desempenho específico $^{[5-6]}$.

Diferenças estruturais entre essas borrachas como, por exemplo, o alto grau de insaturação da fase NR, acarretam em redução na tensão interfacial, levando à incompatibilidade estrutural da mistura NR/SBR. No intuito de melhorar as propriedades mecânicas da mistura NR/SBR várias estratégias tem sido aplicadas, como a adição de diferentes tipos de cargas como forma de melhorar as propriedades mecânicas ${ }^{[7]}$, ou a utilização de técnicas de compatibilização, visando o aumento da adesão entre as fases ${ }^{[8]}$.

No presente estudo esses dois elastômeros foram misturados nas proporções 75:25 e 50:50 em massa. Tanto a mistura dos dois elastômeros quanto a incorporação dos demais ingredientes da formulação foram realizadas de quatro maneiras distintas. A partir das composições obtidas foi investigado o efeito do modo de mistura sobre as propriedades mecânicas.

\section{Experimental}

Os materiais usados neste trabalho foram: borracha natural (viscosidade Mooney 88 ML $1+4\left(100{ }^{\circ} \mathrm{C}\right)$, elastômero SBR (viscosidade Mooney 51,2 ML $1+4\left(100{ }^{\circ} \mathrm{C}\right)$, estireno combinado 22,7\%), óxido de zinco (ativador), ácido esteárico (ativador), enxofre (agente de vulcanização), N-terc-butil-2-benzotiazol-sulfenamida (TBBS, acelerador de vulcanização), aminox, produto da reação, a baixa temperatura, de difenilamina e acetona (antioxidante). Todos foram usados como recebidos.

As formulações utilizadas na preparação das misturas são apresentadas na Tabela 1 (em phr).

As misturas foram preparadas em misturador de cilindros Berstorff, a $50{ }^{\circ} \mathrm{C}$, empregando quatro modos diferentes para a incorporação dos aditivos:

C751 e C501: Os aditivos, com exceção do acelerador, foram misturados inicialmente com a NR e, após a homogeneização, a borracha SBR foi adicionada. Por último, $\mathrm{o}$ acelerador foi acrescentado à mistura homogênea.

C752 e C502: Os aditivos, com exceção do acelerador, foram misturados inicialmente com a SBR e, após a homogeneização, a borracha NR foi adicionada. Por último, $\mathrm{o}$ acelerador foi acrescentado à mistura homogênea.

C753 e C503: As borrachas foram previamente misturadas, antes da adição dos aditivos.

C754 e C504: A cada uma das borrachas foi adicionada metade da quantidade de cada um dos aditivos, com exceção do acelerador. As composições das duas borrachas foram misturadas e, por último, o acelerador foi acrescentado à mistura homogênea.

Para fins de comparação, composições com cada uma das borrachas isoladamente foram também preparadas.

Após a etapa de obtenção das composições no misturador de cilindros, procedeu-se à determinação dos parâmetros reométricos utilizando reômetro de disco oscilatório, da marca Tecnologia Industrial, modelo TI-100, operando com $\operatorname{arco~de~} 1^{\circ}$ e temperatura de $160{ }^{\circ} \mathrm{C}$.

Os ensaios de tração e rasgamento foram realizados em dinamômetro Instron, segundo as normas ASTM D-412 e D-624, respectivamente, a temperatura ambiente, com velocidade de deformação de $500 \mathrm{~mm} / \mathrm{min}$. Para o ensaio de rasgamento foram usados corpos de prova modelo $\mathrm{C}$. Os corpos de prova foram extraídos de placas vulcanizadas a partir dos valores de $t_{90}$ (tempo de vulcanização a $90 \%$ de cura). A dureza Shore A foi determinada seguindo-se o procedimento da norma ASTM D-2240. Os valores das propriedades foram obtidos considerando-se a mediana de, pelo menos, cinco determinações.

\section{Resultados e Discussão}

Apesar de possuir excelentes propriedades elásticas a NR apresenta algumas deficiências, que podem ser solucionadas através da mistura com outros elastômeros. A adição do copolímero de estireno-butadieno (SBR) à borracha natural (NR) é feita por diversos motivos tais como redução no custo do produto final, facilitação na obtenção dos artefatos e por sua alta capacidade de absorver cargas minerais. No entanto, essa incorporação pode resultar em prejuízo às propriedades da mistura, uma vez que os demais ingredientes da formulação poderão estar distribuídos de maneira não uniforme em cada uma das fases.

Os resultados das propriedades mecânicas das misturas com composição 75:25 NR/SBR podem ser vistos na Figura 1, para cada uma das borrachas isoladamente e para as quatro misturas.

A Figura 1 mostra que os valores de dureza para as misturas são intermediários aos das borrachas puras e não variam significativamente com o modo de preparo das misturas, como já era esperado.

Observando-se os valores de resistência à tração e ao rasgamento, apresentados na mesma figura, verifica-se que 
Tabela 1. Formulações das misturas NR/SBR.

\begin{tabular}{lcccccccc}
\hline \multicolumn{1}{c}{ Componentes } & C751 & C752 & C753 & C754 & C501 & C502 & C503 & C504 \\
\hline NR & 75 & 75 & 75 & 75 & 50 & 50 & 50 & 50 \\
SBR & 25 & 25 & 25 & 25 & 50 & 50 & 50 & 50 \\
ZnO (óxido de zinco) & 3 & 3 & 3 & 3 & 3 & 3 & 3 & 3 \\
Ácido Esteárico & 2 & 2 & 2 & 2 & 2 & 2 & 2 & 2 \\
Aminox (antioxidante) & 2,5 & 2,5 & 2,5 & 2,5 & 2,5 & 2,5 & 2,5 & 2,5 \\
Enxofre & 1,5 & 1,5 & 1,5 & 1,5 & 1,5 & 1,5 & 1,5 & 1,5 \\
TBBS (t-butil-2-benzotiazol sulfenamida) & 1,1 & 1,1 & 1,1 & 1,1 & 1,1 & 1,1 & 1,1 & 1,1 \\
\hline
\end{tabular}

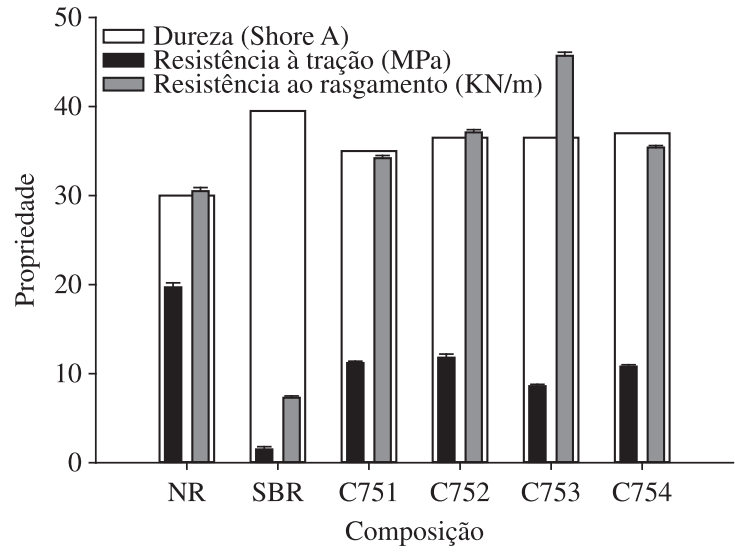

Figura 1. Propriedades mecânicas de misturas 75:25 NR/SBR.

os comportamentos das misturas, com relação a essas duas propriedades, não são iguais. Considerando-se a resistência a tração, comprova-se que o valor desta propriedade para a NR é muito superior ao do SBR e que, para as misturas, os valores são intermediários aos apresentados pelas duas borrachas isoladas. Estes valores, no entanto, dependem do modo de preparo. Para C751 e C752 os valores são próximos e o pior resultado é dado pela composição C753.

$\mathrm{Na}$ Figura 1 observa-se também que, apesar da composição NR ter valor de resistência ao rasgamento bem superior à SBR, a presença desta última na mistura, ainda que em menor proporção, resultou em melhoria na propriedade. Pode ser visto que todas as misturas apresentam valores de resistência ao rasgamento superiores ao da NR e o melhor resultado foi obtido para C753, cujo aumento foi de cerca de $50 \%$, quando comparado com a NR.

A Tabela 2 mostra os parâmetros reométricos para as borrachas puras e para as quatro misturas de 75:25 NR/SBR. Pelos valores de $t 90$ pode ser visto que a NR vulcaniza muito mais rápido que a SBR. Já as 4 misturas apresentam valores de 190 intermediários aos das composições com as borrachas isoladas, porém mais próximos à $\mathrm{NR}$, uma vez que este elastômero está presente em maior proporção. O menor valor de t90 é apresentado pela composição C751, como já era esperado, pois nesta composição o sistema de vulcanização é adicionado na fase NR, enquanto que o maior valor é apresentado pela composição C752, o que também já era esperado, uma vez que a borracha natural vulcaniza mais rapidamente que a borracha SBR.
Tabela 2. Propriedades reométricas de misturas 75:25 NR/SBR.

\begin{tabular}{cccccc}
\hline Material & $\begin{array}{c}\text { t90 } \\
\text { (minutos) }\end{array}$ & $\begin{array}{c}\text { ts1 } \\
\text { (minutos) }\end{array}$ & $\begin{array}{c}\text { Ml } \\
\text { (lb.in) }\end{array}$ & $\begin{array}{c}\text { Mh } \\
\text { (lb.in) }\end{array}$ & $\begin{array}{c}\text { (Mh - Ml) } \\
\text { (lb.in) }\end{array}$ \\
\hline NR & 6,6 & 4,8 & 2,1 & 18,7 & 16,6 \\
SBR & 28,2 & 13,8 & 3,6 & 21,1 & 17,5 \\
C751 & 8,9 & 5,8 & 1,8 & 22,8 & 21 \\
C752 & 10,3 & 6,3 & 3,1 & 23,5 & 20,4 \\
C753 & 9,9 & 6,1 & 3 & 23,3 & 20,3 \\
C754 & 9,6 & 5,8 & 3,3 & 23,5 & 20,2 \\
\hline
\end{tabular}

t90: tempo ótimo de cura; $\mathrm{Ml}$ : torque mínimo; $\mathrm{Mh}$ : torque máximo; e ts1: tempo de pré-vulcanização.

O tempo de pré-cura, ts1, é o tempo disponível durante o qual o material pode ser processado antes que um número significativo de ligações cruzadas tenha sido formado. Pelos dados da Tabela 2, observa-se que os valores obtidos para as misturas são próximos uns dos outros e intermediários aos valores apresentados pelas borrachas isoladas, mostrando que a SBR, ainda que em menor quantidade, aumenta o tempo de segurança para o processamento. Já o torque mínimo, Ml, relacionado com a viscosidade das composições não vulcanizadas, em comparação com a NR teve os valores aumentados com a adição de SBR, excetuando-se C751, já que a borracha SBR é mais viscosa na temperatura de vulcanização.

Com relação ao torque máximo, Mh, as misturas apresentaram valores maiores que as borrachas isoladas, o que indica que a presença de SBR não inibe a vulcanização da NR.

Essas observações podem ser confirmadas pelas curvas da Figura 2, que mostra a evolução do torque em função do tempo durante o processo de vulcanização. A Figura 2 mostra também a tendência da NR à reversão, pois para a composição com esta borracha isoladamente o torque máximo, após atingido, começa a decair, evidenciando a pouca resistência deste elastômero à degradação em altas temperaturas. É interessante notar o caráter protetor do SBR pois embora esteja presente em menor proporção, sua presença inibe o processo de envelhecimento da fase NR nas misturas.

Resultados de propriedades mecânicas das misturas com composição 50:50 NR/SBR podem ser vistos nas Figura 3 para cada uma das borrachas isoladamente e para as quatro misturas.

A Figura 3 mostra que os valores de dureza para as misturas são intermediários aos das borrachas puras e não variam significativamente com o modo de preparo das misturas. $\mathrm{O}$ 


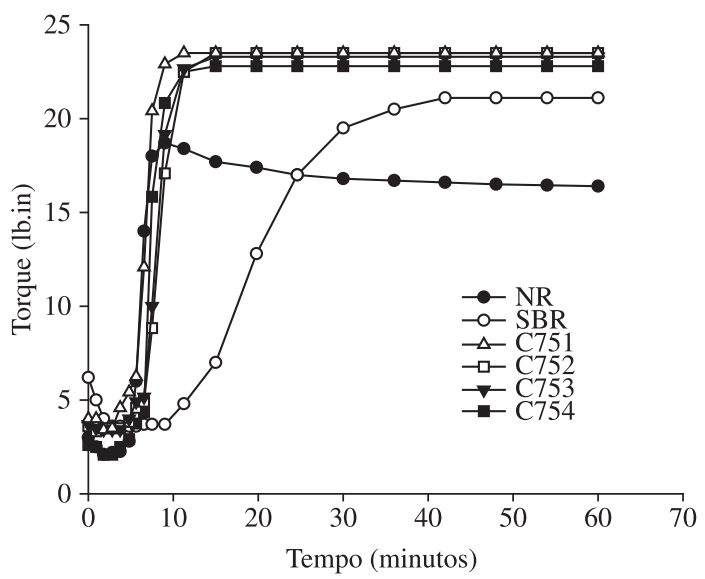

Figura 2. Curvas reométricas de misturas 75:25 NR/SBR.

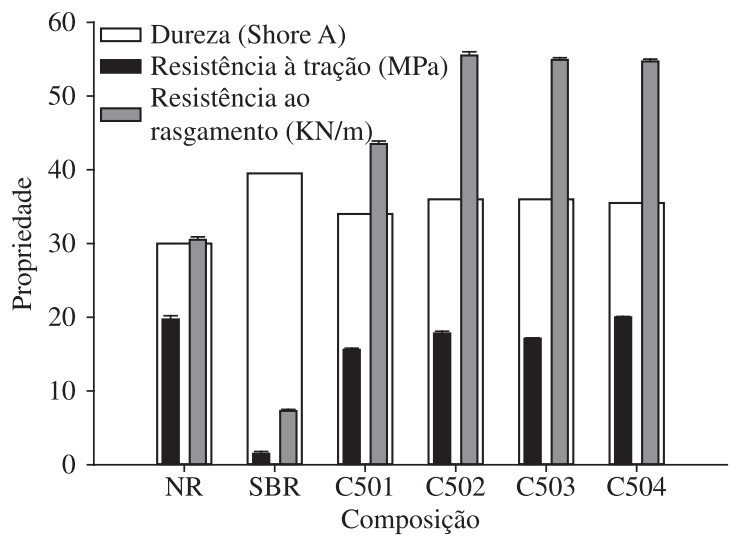

Figura 3. Propriedades mecânicas das misturas 50:50 NR/SBR.

aumento no teor de SBR na composição não alterou de forma significativa os valores de dureza das quatro misturas.

$\mathrm{Na}$ mesma figura pode ser visto também que todas as 4 misturas apresentaram valores de resistência ao rasgamento superiores à NR. A composição C501 foi a de resultado menos expressivo entre as misturas com composição 50:50 NR/SBR, mostrando que a adição dos ingredientes na fase NR, em particular dos agentes vulcanizantes, promove cura excessiva, aumentando a tendência à degradação desta fase, levando a menores valores da propriedade. As demais misturas apresentaram valores parecidos, sendo a C502 ligeiramente superior. O aumento no teor de SBR na composição, de 25 para $50 \mathrm{phr}$, promoveu um aumento na resistência ao rasgamento nas quatro misturas.

Os resultados de resistência à tração para as composições de 50:50 NR/SBR apresentam valores intermediários aos das duas borrachas isoladas. Estes valores, no entanto, dependem do modo de preparo. A resistência à tração para C502 é maior que para C501 mostrando que o aumento da concentração do sistema de vulcanização em favor da fase SBR promove um aumento nesta propriedade. O pior resultado de resistência à tração está associado à mistura C501, onde todos os ingredientes foram adicionados na fase NR e o melhor resultado foi dado por C504. Nesta última composição, metade das quantidades de cada aditivo foi adicionada a cada uma das borrachas, antes da mistura final. $\mathrm{O}$ alto valor dessa propriedade mostra que a vulcanização da fase NR é realmente necessária para se obter boa resistência à tração. Porém a cura excessiva, caso de C501, é prejudicial. O aumento no teor de SBR na composição, de 25 para $50 \mathrm{phr}$, promoveu um aumento na resistência à tração nas quatro misturas, mais uma vez sugerindo a função protetora que esta borracha desempenha sobre a NR.

A Tabela 3 mostra os parâmetros reométricos para as borrachas puras e para as quatro misturas de 50:50 NR/SBR. As curvas reométricas são apresentadas na Figura 4.

Em comparação com as misturas 75:25 NR/SBR, o aumento no teor de SBR na composição promoveu um aumento no tempo ótimo de cura e no tempo de prévulcanização. Nas misturas com composição 50:50 NR/SBR, os parâmetros reométricos não sofreram influência do modo de preparo, com exceção da composição C501.

Os valores mais baixos de resistência à tração e ao rasgamento apresentados pela mistura C501 é possivelmente consequiência da alta concentração de aditivos presentes na fase NR. Como pode ser visto na Tabela 3, o tempo ótimo de cura para esta composição é de 13,8 minutos, mais que o dobro do $t 90$ da NR pura (6,6 minutos). O aquecimento prolongado ao qual ficou sujeita a composição favoreceu a oxidação da fase NR, acarretando baixas propriedades.

A degradação termo-oxidativa das borrachas ocorre por dois mecanismos ${ }^{[5]}$. O primeiro deles passa pela cisão ou despolimerização do elastômero, o que torna o elastômero gradativamente mais macio e pegajoso. No segundo mecanismo, a oxidação das ligações duplas carbono-carbono dá origem a radicais livres que, por sua vez, podem atacar as cadeias do polímero, levando a um aumento no número de ligações cruzadas. Neste trabalho, as misturas com composição 75:25 NR/SBR apresentaram valores de torque máximo, relacionado ao número de ligações cruzadas, superiores aos das borrachas isoladas. Entretanto, as misturas com composição 50:50 NR/SBR apresentaram valores de torque máximo semelhantes aos das borrachas isoladas. Observa-se também, a partir das Figuras 2 e 4, que o tempo ótimo de vulcanização para a NR foi menor que para todas as misturas e bem menor que para a SBR. Isso significa que os tempos ótimos de cura $\left(\mathrm{t}_{90}\right)$ usados na vulcanização das misturas são excessivamente altos, considerando-se a fase NR, podendo então provocar degradação parcial desta fase. Entretanto, como não se observa queda no torque máximo, pode-se supor que ou a fase NR está sofrendo degradação via o segundo mecanismo, ou estaria havendo, de certa forma, uma ação protetora do SBR que, nas misturas, dificultaria a degradação da NR, ou ambos os casos. De acordo com a literatura ${ }^{[9]}$, a degradação da NR acontece segundo o primeiro mecanismo (cisão/despolimerização), presume-se que nos casos em que a vulcanização da NR é fortemente favorecida, os produtos da cisão possam estar auxiliando na vulcanização do SBR. Entretanto, a vulcanização excessiva da NR pode levar a um grau de degradação desta borracha, comprometendo o desempenho mecânico da mistura. 
Tabela 3. Propriedades reométricas de misturas 50:50 NR/SBR.

\begin{tabular}{cccccc}
\hline Material & $\begin{array}{c}\text { t90 } \\
\text { (minutos) }\end{array}$ & $\begin{array}{c}\text { ts1 } \\
\text { (minutos) }\end{array}$ & $\begin{array}{c}\text { Ml } \\
\text { (lb.in) }\end{array}$ & $\begin{array}{c}\text { Mh } \\
\text { (lb.in) }\end{array}$ & $\begin{array}{c}\text { (Mh - MI) } \\
\text { (lb.in) }\end{array}$ \\
\hline NR & 6,6 & 4,2 & 2,1 & 18,7 & 16,6 \\
SBR & 28,2 & 12 & 3,6 & 21,1 & 17,5 \\
C501 & 13,8 & 7,6 & 2,3 & 19 & 16,7 \\
C502 & 17,2 & 9,7 & 3,5 & 20,3 & 16,8 \\
C503 & 16,2 & 9,2 & 3,1 & 20,4 & 17,3 \\
C504 & 16,4 & 9,4 & 3,2 & 20,3 & 17,1 \\
\hline
\end{tabular}

t90: tempo ótimo de cura; Ml: torque mínimo; Mh: torque máximo; e ts1: tempo de pré-vulcanização.

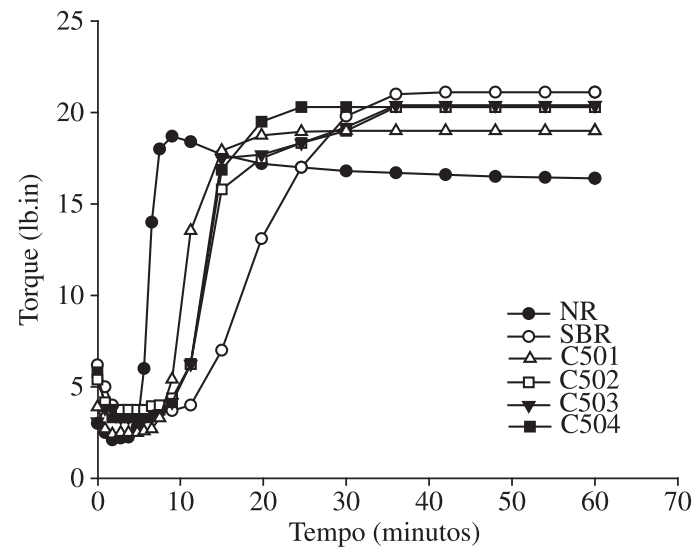

Figura 4. Curvas reométricas de misturas 50:50 NR/SBR.

Dessa maneira, mesmo tendo a borracha natural propriedades bem superiores à SBR, quanto menos se favorece a vulcanização da fase NR nas misturas, melhores deverão ser os resultados mecânicos. Logo, a composição C501, com 50 phr de NR, apresentou melhores resultados que a composição C751, que contém um teor mais elevado de NR. Esperava-se que os resultados de propriedades mecânicas para as composições C751 e C753 como, por exemplo os de resistência ao rasgamento, fossem mais baixos, quando comparados às demais composições 75:25 NR/SBR, já que tanto em C751 quanto em C753 a fase NR possui uma alta concentração de aditivos, isto é, na primeira composição os aditivos são incorporados na fase $\mathrm{NR}$, na outra, os aditivos migram preferencialmente para a fase NR, uma vez que possuem maior afinidade por esta fase. Porém, na composição C751 o tempo ótimo de cura de 8,9 minutos, provavelmente não foi longo o suficiente para promover uma grande oxidação da fase NR. Além disso é preciso lembrar que as quantidades de todos os aditivos foram mantidas constantes, independente da formulação, o que significa que, para o mesmo teor de agentes de vulcanização, havia na mistura C751 uma quantidade bem maior de NR e o excesso dessa borracha poderia estar compensando a parte degradada.

Nas misturas com composição 50:50 NR/SBR, os maiores tempos ótimos de cura provavelmente promoveram a oxidação da fase NR, confirmando os baixos resultados de resistência à tração para as composições C501 e C503, como esperado ${ }^{[10]}$. No entanto, os resultados mecânicos para essas composições, tanto de resistência a tração quanto resistência ao rasgamento, foram superiores aos das composições 75:25 NR/SBR, mostrando a importância do caráter protetor do SBR.
A incorporação da SBR ao elastômero NR foi vantajosa do ponto de vista das propriedades mecânicas, pois a sua presença, mesmo em quantidades menores, ofereceu proteção contra a tendência à reversão da NR.

\section{Conclusão}

A maneira como os aditivos são incorporados em misturas de borrachas influencia suas propriedades mecânicas. Se os componentes elastoméricos têm características muito diferentes, como é o caso da mistura NR/SBR, as melhores propriedades são obtidas favorecendo-se a vulcanização da borracha que, quando isolada, apresenta propriedades inferiores, ao mesmo tempo em que se procura preservar as boas qualidades da outra.

\section{Agradecimentos}

Os autores expressam seus agradecimentos ao Conselho Nacional de Desenvolvimento Científico e Tecnológico CNPq e à Coordenação de Aperfeiçoamento de Pessoal de Nível Superior - CAPES pelo apoio financeiro.

\section{Referências Bibliográficas}

1. Boochthum, C. K. \& Prajudtake, W. - Eur. Polym. J., 37, p.417 (2001).

2. El-Sabbaagh, S. H. - Polym. Testing, 22, p.93 (2003).

3. Visconte, L. Y.; Costa, H. M.; Nunes, R. C. R \& Furtado, C. R. G. - Polímeros - Cienc Tecnol., 13, p.125 (2003).

4. Mangajah, D. - Rubb. Chem. Technol., 75, p.365 (2002).

5. Brydson, J. A. - “Natural Rubber”, in: Rubbery Materials and their Compounds, Elsevier Applied Science, London (1988).

6. Brydson, J. A. - "Styrene-Butadiene Rubbers" in: Rubbery Materials and their Compounds, Elsevier Applied Science, London (1988).

7. Findik, F.; Yilmaz, R. \& Köksal,T. - Mater. Des., 25, p.269 (2004).

8. Ismail, H. \& Hairunezam, H. M. - Eur. Polym. J., 37, p.39 (2001).

9. Morton, M. - "Introduction to Polymer Science", in: Rubber Technology, M. Morton (ed.), Van Nostrand Reinhold, New York (1987).

10. Visconte, L. Y.; Martins, A. F.; Nunes, R. C. R. \& Furtado C. R. G. - Polímeros - Cienc Tecnol., 11, p.76 (2001).

Enviado: 08/01/09

Reenviado: 01/10/09

Aceito: $11 / 10 / 09$ 